\title{
Sur la recherche en Europe
}

Je commencerai par quelques rappels historiques, qui me paraissent éclairer la situation actuelle de la recherche en Europe.

Avant-guerre, il n'est guère contestable que l'Europe assure, en physique et chimie notamment, un leadership mondial. Après 1945, l'Europe est très affaiblie et divisée en deux blocs. Grâce à la prise de conscience d'hommes politiques, à la mobilisation de hauts fonctionnaires et de scientifiques, une dynamique de construction de l'Europe économique et politique, mais aussi scientifique, se met en place.

Dans un premier temps, l'avènement de la physique nucléaire dans les années 30 rendait nécessaire la construction de grands équipements, très coûteux, pour lesquels une mutualisation s'imposait. C'est ainsi que le CERN $^{(1)}$ fut créé en 1954, à l'initiative de 12 États. Il fut le prototype et l'inspirateur de tout un ensemble d'instituts et de grands instruments créés par la suite, tels l'ESO dans le domaine de l'astronomie, installant ses grands télescopes au Chili, l'ILL et l'ESRF implantés à Grenoble. Sans oublier l'Euratom, créé en 1957, et dont la réalisation majeure, le JET, a donné à l'Europe et à la France la légitimité d'accueillir ITER.

Mais la physique nucléaire n'est pas le seul enjeu auquel l'Europe a dû répondre, notamment en termes de compétition et de coopération avec les États-Unis. Un autre grand défi a été l'espace, défi scientifique mais aussi technologique, qui concerne aussi bien les lanceurs que les satellites : la France créa le CNES en 1961, et les États européens l'ESA en 1975.

C'est ainsi qu'en l'espace de 20 ans, ont été créés des centres d'excellence, rivalisant avec les États-Unis au niveau mondial, et porteurs d'une dynamique scientifique exceptionnelle. Ceci a permis à la recherche fondamentale en Europe d'être au même niveau, dans les domaines concernés, que celle des États-Unis.

Mais une autre impulsion va mettre progressivement en place en Europe un ensemble de programmes de recherche liés à la compétition économique. Il s'agit des programmes cadres PCRD et EUREKA.
Schématiquement, il est alors acté que l'Europe est en phase de rattraper les États-Unis, et, que si ce rattrapage est assez rapide de 1945 à 1980, il s'est ensuite essoufflé ; certains pays vont même connaître, après les années 90 , un décrochage en termes d'innovation. Cet état de fait aboutit à ce que l'on appelle la "stratégie de Lisbonne", définie en 2000 , et qui vise à faire de l'Europe la «société de la connaissance ». Conscients dès les années 60 que la science et la technologie sont une des bases de la croissance économique et du progrès social, les responsables européens vont mettre en place, sous l'impulsion du Commissaire Philippe Busquin, ce qu'on appellera plus tard l' «Espace européen de la recherche ". La commission, qui aura l'initiative des PCRD, présente dès 1969 quarante-sept propositions dans sept thématiques qui reflètent les préoccupations du moment : informatique, télécommunications, transport, océanographie, métallurgie, pollutions et météorologie. En 1984 est institué le premier programme cadre, et cette maturation s'achève par l'inscription de la recherche-développement comme domaine de compétence partagée dans l'acte unique.

\section{Quels sont les objectifs de ces programmes?}

- Premier objectif : favoriser l'intégration des systèmes de recherche nationaux par la mise en réseau de laboratoires publics ou privés autour de projets ciblés.

- Deuxième objectif : favoriser la constitution de centres d'excellence de niveau mondial. Mais ces objectifs sont sous contrainte. En effet, le principe de ces programmes est la recherche précompétitive afin de ne pas distordre la concurrence. Il s'agit de ne pas financer ce qui peut être interprété comme une aide directe à l'industrie. L'on suit donc une logique top-down, pilotée par la Commission. Cette contrainte n'existait pas lors de la première phase de la construction de l'Europe de la recherche, à l'exception du cas de l'ESA, ce qui n'est d'ailleurs pas resté sans poser problème.

Parallèlement, les grands industriels s'allient pour faire front à la concurrence ; 
